\title{
La aplicación de la Ley de Orgánica de Comunicación y la democratización de frecuencias para medios televisivos en Ecuador
}

\section{The application of the Communication Organic Law and the democratization of frequencies for television media in Ecuador}

\author{
Del Pozo Raymond, Víctor Hugo ${ }^{1 *}$, Llumiguano Huacon, Shirley Gillian ${ }^{1}$ y Noboa Auz, Marjorie \\ ${ }^{1}$ Universidad de Guayaquil \\ *victor.delpozor@ug.edu.ec
}

DOI: https://doi.org/10.26871/killkana_social.v2i1.188

\begin{abstract}
Resumen
En la actualidad se observa que los medios de comunicación ecuatorianos se encuentran regidos por la Ley Orgánica de Comunicación, en donde las frecuencias del espectro radioeléctrico pertenecen al estado y son reguladas por la Agencia de Regulación y Control de las Telecomunicaciones (ARCOTEL). Luego de establecida la Constitución del 2008, fue instituida la igualdad en acceso a la información sin importar etnia, raza, o condición social. Esta institución gubernamental nace como iniciativa para regular las comunicaciones debido a que hasta el año 2012 los medios privados, al no existir un marco legal que fomente una distribución equitativa de las frecuencias para medios audiovisuales, se podía incurrir a intereses propios o a favores políticos al momento de dicha adjudicación, por lo cual se inicia un proceso en el cual se cambia la forma de asignar estas frecuencias. Mediante esta investigación se buscó analizar comparativamente el proceso de la distribución de frecuencias del espectro radioeléctrico para medios audiovisuales con los lineamientos legales antes y después de la aplicación de la Ley Orgánica de Comunicación (LOC).
\end{abstract}

Palabras clave: democratización, frecuencias radioeléctricas, medios audiovisuales, comunicación.

\begin{abstract}
Nowadays it is observed that the Ecuadorian media are governed by the Organic Law of Communication in which the frequencies of the radio-electric spectrum belong to the state and are regulated by the Agency for the Regulation and Control of Telecommunications (ARCOTEL by its Spanish abbreviation). After the establishment of the 2008 Constitution, equal access to information was established as a right regardless of ethnicity, race, or social status. This governmental institution was created as an initiative to regulate communication, because until 2012, private media, in the absence of a legal framework to encourage an equal distribution of frequencies for audiovisual media, favored self-interests as well as political favors at the time of assigning these frequencies. Therefore, a process of change for the way in which these frequencies are assigned has been initiated. The purpose of this research was to compare the process of frequency distribution of the radio-electric spectrum for audiovisual media with the legal guidelines before and after the application of the Organic Law of Communication (LOC).
\end{abstract}

Key words: Democratization, radio frequencies, audiovisual media, communication.

\section{Introducción}

Una frecuencia del espectro radioeléctrico hace posible que la comunicación se lleve a cabo en donde a través de esta se pueda informar, educar y entretener a un habitante. Es aquí donde nace la importancia de la realización de un estudio acerca de su distribución, debido a que la asignación equitativa de autorizaciones para funcionamiento de medios audiovisuales puede aportar al desarrollo social y cultural de un país.
La presente investigación busca establecer el estado en que sea democratizado la asignación de frecuencias para medios audiovisuales. La importancia de una correcta democratización se podrá plasmar en el libre acceso a la información y comunicación que todo ciudadano pueda tener en una sociedad. Esta es la razón por la que se compara el antes y después de la aplicación de la LOC en los aspectos relacionados en la forma de adjudicar una frecuencia. 
Mediante la Declaración de los Derechos Humanos, se estableció un instrumento internacional que promueve el derecho a la libertad de expresión y opinión de toda persona. Por esta razón, la democratización de la distribución de frecuencias permite el libre acceso a la información a todo ciudadano. Un medio de comunicación debe nacer con el fin de entretener e informar a una comunidad, pero el canal de televisión en su gran mayoría pertenece al sector privados y son estos quienes defienden sus intereses económicos. La globalización y el deseo de rentabilidad ocasionó que estos se preocupen por transmitir contenidos que vendan y no necesariamente sean los que urgen dentro de una nación.

Al momento en entro en vigencia la Constitución del 2008 esta garantiza la igualdad de acceso a la comunicación para todas las personas sin importar condición social, etnia o raza por lo que se abrió la puerta a la creación de un nuevo espacio para medios comunitarios y públicos.

En la actualidad la democratización de la distribución de frecuencias de medios audiovisuales permite al ciudadano de tener diferentes puntos de vista acerca de una noticia relevante para la sociedad, generando la posibilidad de defender sus derechos ante cualquier irregularidad que se pueda presentar en su comunidad.

El hecho de analizar comparativamente la distribución de frecuencias del espectro radioeléctrico de los medios audiovisuales permite conceptualizar los elementos principales que se vinculan al espectro radioeléctrico de medios audiovisuales y así determinar las características principales de esta distribución.

El diseño de investigación busca describir y explicar los elementos que forman parte de la distribución de frecuencias además de su historia y evolución.

La comunicación televisiva es una de las más utilizados por la ciudadanía debido su cobertura nacional y bajo costo. Este medio audiovisual es uno de los más influyentes a la hora de la construcción de la opinión pública debido a lo masivo que este tipo medio puede llegar a ser y así del porque se puede informar a gran parte de ciudadanos.

La televisión suele captar al televidente gracias al formato de audio y video. Esta hace que sea uno de los medios mayormente consumido por un ciudadano al momento de buscar información, sus presentaciones de mensajes comunicacionales convencen a la audiencia de temas políticos, culturales, sociales, religiosos o culturales.

La televisión construye una puesta en escena discursiva de los fenómenos que son lo cotidiano de la información a partir de elecciones que conducen a una forma verbal y visual que da sentido y produce representaciones colectivas que permiten que se puedan inteligibilizar en el mundo. (Antezana, 2011, pág. 107)La televisión ingresa en la mente de las personas y es el medio que se impone a pesar de tener nuevos sistemas de comunicación como: videos juegos, redes sociales, plataformas visuales, base de datos de CDROM o los software educativos (Liceras, 2013, pág. 355).
El medio de comunicación ha sido popular durante años llegando a competir con la educación que se da en casa o en la escuela debido a que este genera impactos en lo social, cultural e incluso facilita la socialización a las nuevas generaciones. (Valle, Denegri, y Chávez, 2012, pág. 184)

Muchos piensan que no existe una retroalimentación directa del receptor dentro de la comunicación televisiva, pero mediante está el receptor recibe el mensaje, lo decodifica y lo difunde a otras personas además de tener la opción de expresar su criterio sobre el contenido observado lo que puede afectar a una colectividad.

El contenido transmitido a través de la pantalla chica tiene diferentes formatos a diferencia de la radio, prensa escrita o los medios digitales. En la programación que emite un este medio audio visual de este tipo puede ir desde anime, series televisivas hasta informativos.

El paso de los años desde la creación de la televisión permite que se generen nuevos contenidos visuales que atrapen más espectadores. El cambio cultural que surge en cada individuo incita a la hibridación de los programas transmitidos por los canales audiovisuales permitiendo una mayor oferta y demanda.

El manejo de la programación en medios de comunicación se debe al rating que tenga cada contenido audiovisual, si uno de los programas no es muy consumido es quitado de las diferentes franjas horarias para ubicar otro que aumente el nivel de audiencia del canal.

Según (Llumiguano Huacón, 2017) el espectro radioeléctrico son ondas electromagnéticas o también ondas hertzianas que viajan por el aire y transmiten una señal de audio y video a diferentes decodificadores como son los aparatos de radio, televisión u otros equipos que pueden percibir la señal. Estas ondas pueden transmitir una diversidad de contenidos audio visuales debido a su naturaleza. Para los medios televisivos estas frecuencias se dividen VHF y UHF la cuales en la actualidad son propiedad del estado ecuatoriano y a su vez reguladas por la Agencia de Regulación y Control de las Telecomunicaciones (ARCOTEL) la cual se encarga de la concesión de frecuencias en el país.

En el proceso de distribución de frecuencias se contempla el $33 \%$ son para medios públicos, $33 \%$ para medios privados y el $34 \%$ para medios comunitarios, en base a esta normativa se debe realizar un concurso donde cualquier persona, ya sea natural o jurídica, puede acceder a una frecuencia electromagnética.

El beneficio que un ciudadano puede tener a partir de la democratización de las frecuencias es el hecho de tener el poder de decisión y la soberanía al obtener opciones de medios audiovisuales que puedan representar los derechos de una comunidad.

Uno de los graves problemas para el ejercicio de la libertad de expresión es la concentración de la propiedad de los medios en pocos grupos familiares. Esta situación imposibilita la real democratización de sus paquetes accio- 
narios de tal forma que las políticas comunicacionales y los controles internos no dependan de la voluntad omnímoda de un solo dueño sino del criterio debatido y consensuado de una junta de propietarios. (Vallejo, 2011)

En Ecuador por varias décadas las comunicaciones televisivas estuvieron privatizadas y ningún medio audio visual comunitario era reconocido legalmente dentro de esta sociedad. Por esta razón, la democratización permite el reconocimiento de medios privados, públicos y comunitarios generando mayores posibilidades de acceso a productos audio visuales a los ecuatorianos.

\subsection{Proceso de concesión previo a la LOC}

Este proceso constaba de los siguientes requisitos:

1) Nacionalidad del concesionario, acreditada de acuerdo con la Ley.

2) Escritura pública de constitución de la sociedad concesionaria y título de propiedad de los equipos.

3) Lugar en que la estación será instalada.

4) Nombre de la estación radiodifusora o televisora, potencia de operación, frecuencia asignada, horario de trabajo y el indicativo que utilizará para identificarse.

5) Garantía a favor del Instituto Ecuatoriano de Telecomunicaciones.

6) Cantidad que pagará mensualmente por la utilización de la frecuencia.

Para poder adquirir una frecuencia del espectro radio eléctrico se debe de pasar por las siguientes etapas:

1) Entrega de documentación de requisitos física y por medio magnético a la Arcotel.

2) La Agencia de Regulación y Control de Telecomunicaciones deberá remitir los cinco mejores expedientes de los participantes al Consejo de Regulación de la Información y Comunicación.

3) La CORDICOM procede a revisar los proyectos remitidos.

4) Arcotel recibe el informe final de la Cordicom para luego proceder a dar el permiso a los participantes ganadores del concurso.

Los parámetros que califica la Cordicom son:

1) Promoción de derechos.- igualdad, inclusión, pluriculturalidad, protección a los grupos de atención prioritaria; esto equivale al $5 \%$ de la calificación y hasta 40 puntos.

2) Participación.- contenidos formativos y educacionales donde promuevan a la participación del ciudadano, este tiene un valor del $5 \%$ y un puntaje hasta 30.

3) Dialogo.- contenidos que generen la participación de la audiencia en el programa, este equivale hasta 20 puntos y el $5 \%$.

4) Libre caracterización. - exprese su orientación y los objetivos por el cual se desea crear el medio tiene un puntaje de 10 puntos máximo y representa al $0 \%$.

\section{Metodología}

Se utilizó el método histórico - lógico, método descriptivo además de la revisión bibliográfica para establecer la situación actual de la democratización de los medios audiovisuales.

Como criterios tomados dentro de este estudio tenemos:

- Tiempo de concesión

- Requisitos para la concesión de frecuencias

Para la realización de este proceso investigativo se consulto documentación como "La Ley Orgánica de Comunicación de Ecuador, ¿Un avance en el ejercicio efectivo de las libertades de expresión e información y en la participación ciudadana?" de la Revista de Derecho Político, "La participación social en el Estado, una relación complementaria de la Comunicación" de la revista Cuadernos de la Cordicom, entre otros.

Se realizó, además, la revisión de documentos tales como: Ley Orgánica de Comunicación (2013), la Constitución de la República del Ecuador (2008), informes de investigación, así como documentos que recogen datos sobre la adjudicación de frecuencias radio eléctricas para medios audio visuales.

Luego de la triangulación de la información recopilada se procedió a establecer las siguientes dimensiones analíticas:

- Proceso de adjudicación de frecuencias después de la LOC.

- Proceso de adjudicación de frecuencias antes de la LOC.

\section{Resultados}

Se puede observar luego del estudio realizado que antes del 1997 los medios de comunicación estaban regidos por la Ley de Radiodifusión y Televisión y en el cual constaba las personas naturales y jurídicas podían ser propietarios de una frecuencia del espectro radioeléctrico. Se pudo notar que esta concesión era dada por un periodo de 10 años para la transmisión de diferentes programas culturales, educativos, entretenimiento y educativos, al cumplirse este tiempo tenían que renovar el proceso para la obtención de la frecuencia por un periodo igual al anterior y pudiendo tener hasta un $25 \%$ de inversión extranjera. Un medio comunitario en ese marco legal podía tener una señal de onda media donde no existían estaciones regionales o nacionales.

Luego de la (LOC) se hicieron reformas en la forma de adjudicar una frecuencia del espectro radioeléctrico. Entre los cambios que trajo esta es la distribución equitativa del espectro radioeléctrico en donde se establece que el $33 \%$ para medios privados, $33 \%$ públicos y $34 \%$ para la operación de medios comunitarios, además su concesión será renovable cada 15 años.

Del análisis de estos se puede indicar que la entidad encargada de otorgar la concesión antes de la LOC era la CORNATEL en base a los requisitos anteriormente indicados donde no se establece prioridad a la equidad en 
la distribución de frecuencias para canales de televisión además de la necesidad de una tecnificación necesaria a en dicho proceso.

A partir del 2008 se inicia un proceso de licitación para dar a conocer que cuantos medios existían y si estos eran adquiridos conforme a la ley. En este proceso dos canales de televisión de cobertura Nacional pasan a manos del Estado y se crean nuevos medios de comunicación de servicio público.

Una vez aplicada la LOC se procede a dividir e etapas el proceso buscando que este se realice de una manera más técnica con el fin de generar una equidad en la distribución de las frecuencias. Sin embargo, se debe buscar que el proceso se socialice aún más a los ecuatorianos para que se garantice la transparencia del mismo.

\section{Conclusiones}

La aplicación de la LOC ha permitido la generación de nuevos espacios para medios televisivos, pero proceso de concurso para la concesión de una frecuencia audio visual puede ser perfectible profundizando a un más en la equidad debido a que al considerar la proporción asignadas a medios públicos y comunitarios supera sustancial al margen asignado a los medios privado. Además de considerar la complejidad en participación para estos concursos para un medio comunitario. Se puede apreciar que luego de estar establecida la LOC se ha podido frenar varios excesos cometidos por varios medios audio visuales previa a esta ley.

El proceso actual presta facilidades a quienes quieren participar, pero este debe ser público para que todos los ciudadanos y concursantes conozcan quienes van obteniendo mayor puntaje y así se pueda transparentar aún más este proceso que afecta a toda la ciudanía. Desde que entra en vigencia la LOC a junio del 2017 se crean los 5 medios de comunicación comunitarios televisivos que representan un $0.90 \%$ en la señal VHF Y UHF.

En Ecuador la concesión del espectro radioeléctrico es debatida porque este hace que las comunicaciones se realicen a larga de distancias y su distribución antes del 2008 era para medios privados.

\section{Referencias Bibliográficas}

Alonso, M. (2016). "En tu casa o en la mía": la entrevista como infoentretenimiento. Doxa.comunicación, 73 99.

Antezana, L. (2011). La mujer en la televisión: el caso chileno. Cuadernos de Información, 105 - 117.

Arroyo, D. (2011). Desarrollo social y comunicación en América Latina. Resvista Oficios Terrestres, 01 - 15.

Barranquero, A. (2012). De la comunicación para el desarrollo a la justicia ecosocial y el buen vivir. Cuadernos de Información y Comunicación, 63 - 78.

Centeno, J. (2017). Hegemonia Comunicacional y libertad de expresión en Venezuela. En el caso RCTV. Revista Mexicana de Opinión Pública, 35 - 53.
Franco, A. (2015). ¿Cómo afecta el uso de las nuevas tecnologías en los gobiernos modernos? El aumento o la diminución de la opinión pública. Encrucijada. Revista electrónica del centro de Estudios en Admiistración Pública, 01 - 14.

Fuentes, L. (2016). Telerrealidad. El fin de la información en la televisión. Universidad de Sevilla, 1-57.

García, J. (2011). Dimensiones y tipología de las actividades de participación de la audiencia en la televisión pública. Ámbitos, 175 - 195.

Gómez, P. (2013). Libertad de Expresión en México. Revista Méxicana Comunicación, 1 - 4.

Gumucio, A. (2011). Comunicación para el cambio social: clave del desarrollo participativo. Signo y Pensamiento, 26 - 39.

Liceras, n. (2013). La educación informal de los medios de comunicación y protección de los menores de la violencia en televisión: Historia de un Fracaso. Profesorado, 353 - 365.

Llumiguano Huacón, S. G. (2017). Análisis comparativo de los procesos de distribución de las frecuencias del espectro radioeléctrico para medios audiovisuales en ecuador, antes y después de la ley orgánica de comunicación (Tesis Doctoral, Universidad de Guayaquil). Descargado de http: / / repositorio.ug.edu .ec/handle/redug/21108

Magdaleno, A. (2016). La Ley Orgánica de Comunicación de Ecuador, ¿Un avance en el ejercicio efectivo de las libertades de expresión e información y en la participación ciudadana ? Revista de Derecho Político, 291 $-326$.

Mut, M. (2013). Apuntes sobre la opinión pública a pie de calle. Revista de Comunicación de la SEECI, 1 - 10.

Ramirez, S. (2015). Estado de derecho y libertad de expresión. Revista Ciencia Jurídica y Política, 11 22.

Romeu, V. (2011). Diálogo y comunicación intercultural. Pretextos para reflexionar sobre la relación - sujeto en la comunicación humana. Medios y Comunicación de la Universidad de Chile, 1 - 33.

Sáenz, 1. (2016). La participación social en el Estado, una relación complementaria de la Comunicación. Cuadernos del Cordicom, 109 - 134.

Valenzuela, G. (2013). Libertad de expresión y su impacto en la democracia. Jurídicas CUC, 89 - 100.

Valle, C. d., Denegri, M., y Chávez, D. (2012). Alfabetización audiovisual y consumo de medios y publicidad en universitarios de Pedagogía en Chile. Comunicar, $183-191$.

Vallejo, R. (2011, agosto). La Línea de Fuego.

Recibido: 16 de febrero de 2018

Aceptado: 12 de marzo de 2018 